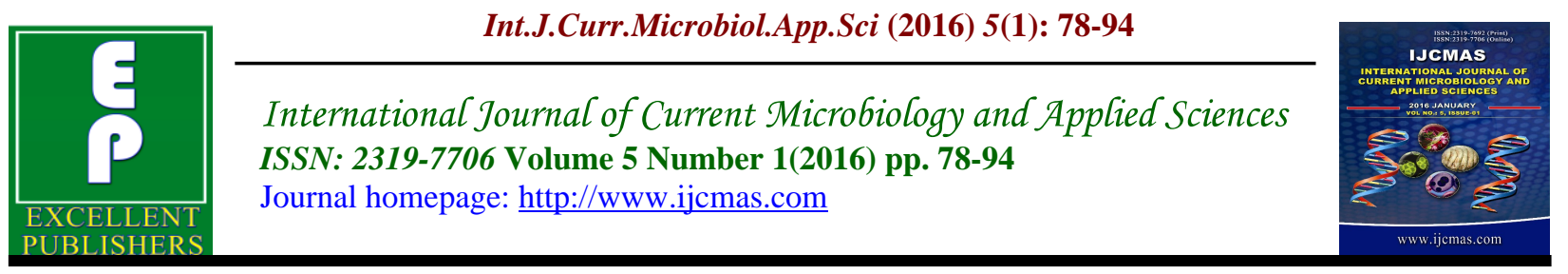

Original Research Article

doi: hhttp:///dx.doi.org/10.20̄0̄

\title{
Phytoplankton and Macrophytic Floral Studies in Kamalapur Reserviour of Karimnagar District of Telangana, India
}

\author{
G. Odelu* \\ Department of Botany, Government Degree College, Jammikunta, Karimnagar, \\ (Satavahana University), Telangana-505122, India \\ *Corresponding author
}

\begin{abstract}
A B S T R A C T
Keywords

Bacillariophyceae,

Kamalapur,

Phytoplankton,

Nitrogen fixing,

Macrophytes

Article Info

Accepted:

22 December 2015

Available Online: 10

10 January 2016

Plankton communities are broadly classified into two basic categories such as phytoplankton and zooplankton. The integration and interaction of different physical, chemical and geo-morphological characteristics of any water body; Biological assessment is a useful alternative in assessing those systems. It is occupied nearly 700 acres and its downstream water utilisation area nearly 2000 acres. Every month collection by early morning 7 am to 11 am and evening $5 \mathrm{pm}$ to $6 \mathrm{pm}$ during research period from June 2013 to May 2015. Macrophytes of study area are 91 species belonging to Monocots, Dicots, Pteridophytes. The algae are totally 56 species of belonging to genera fewer than 4 Classes Bacillariophyceae, Chlorophyceae, Cyanophyceae, Euglenophyceae. Near saturation levels, coinciding with short-term blooms in dry periods and in October indicate dominant photosynthetic activity over respiration. Importantly, though pigments of photosynthesis content in October-November bloom was double to that found during the January-February bloom, the oxygen was clearly under saturated during the former period.
\end{abstract}

\section{Introduction}

Plankton occurs in all natural water as well as in artificial impoundments like ponds, tanks, reservoirs, irrigation cannels and rivers. The plankton study is a very useful tool for the assessment of water quality is any type of water body and also contributes to an understanding of the basic nature and general economy of the water body of river.
Telesh (2004). Plankton communities are broadly classified into two basic categories such as phytoplankton and zooplankton. The phytoplankton organisms are exclusively of plant origin and are thus autotrophy belonging to the first trophic level and being the primary producer from the lowest trophic level in the food chain of freshwater 
ecosystem and play a key role in fish culture. These are minute microscopic plants, passively floating in water often multiply rapidly and give a turbidity to the water. Phytoplankton which includes bluegreen algae, green algae, diatoms, desmids, euglenoids etc. are important among aquatic flora. They are ecologically significant (important) as they form the basic link in the food chain of all aquatic animals, Misra et.al. (2001).

Physico-chemical parameters of any water body though, provide a good indication about the Water chemistry and quality, that alone does not reflect the clear picture of the ecological condition of the water body due to lack of proper integration with ecological factors (Karr et al., 2000). Since a biotic community is the outcome of the integration and interaction of different physical, chemical and geo-morphological characteristics of any water body, biological assessment is a useful alternative in assessing those systems (Stevenson and Pan, 1999). They are mainly responsible for net as well as the gross primary productivity of the system and are the source of base level energy in food webs maintaining in the water bodies.Habitat quality specificity of the different members of phytoplankton is seen to be reflected in their distribution and occurrence in relation to the quality of water where do they live (Bhatt, et al., 1999; Saha et al., 2000). The pond also showed an interesting observation during pre monsoon and monsoon seasons. Higher densities of during these seasons may be attributed for mixing of pond water and mobilisation of nutrients due to rain and surface runoff water that dictates towards the pond. Algal analysis thus showed that water quality of the pond has reached at threshold level and therefore, it need some corrective measures to maintain the water chemistry of the pond to save that historical site heritage from further deterioration.
Considerable work has been done in India about systematic survey, distribution, periodicity and ecology of algae in different habitats (Das et al., 2009, Adhikary et al.2010,Das et al.,2010, Bhakta et al.,2010, Das and Adhikary, 2012a,b\&c and Kumaraswamy et al.2013). Recently Mahajan (2005), Gupta and Anuj Bhadauriya 2007, Roy Zacharias and Joy (2007), Maya Subramoni, 2007, Kavitha and Rajini Balasingh (2007). Latha and Ramachandra Mohan (2010),, Ramadosu and Sivakumar (2010) and Chinnaiah et al.,(2011) studied on various fresh water bodies and described about physicochemical characteristics along with algal population studies. Sreelatha and. Rajalakshmi (2005) studied dynamics of chlorophyceae in river Goutami, Godavari, Yanam, U.T. of Pondicherry; Aijaz et.al. (2009) studied phytoplankton of Wulor Lake (Ramsarsite), Jammu and Kashmir, India. In India the ecological aspects are focus of investigation in a few rivers such as Adyar, Chacko (1954, Coovum, Iyengar and Venkataraman (1951), Hoogly, Roy (1955), Ganga, Lakshminarayan (1965), Moosi Venkateswaralu (1969), Gomati Prasad and Saxena (1980), Vishwamitri, Nandan and Patel (1983; 1984 and 1986) and Godavari, Rajyalakshmi and Premswarup (1975), Yamuna Chakraborthy et.al. (1977). Hence, the present study was undertaken to know the influence of physic-chemical parameters of water on algal populations and their seasonal changes of Kamalapur reservoir. Heterotrophic organisms consume organic carbon to support their metabolic activities.

\section{Study Area}

Kamalapur is belong to district Karimnagar, Telanagana state. It is one of the rural areas of this district bordered with East Warangal district, South Elkathurhy mandal, North the Jammikunta, and West Huzurabad mandal. 
It is occupied nearly 700 acres and its downstream water utilisation area nearly 2000 acres. Its borders with reservoir west Uppal village south Deshraj pally north and east itself. Downstream water combines with near ponds i.e.Vangapally and other small aquatic bodies.

\section{Materials and Methods}

The water samples were collected in polythene containers from four stations of reservoir every month early morning 7 am to $11 \mathrm{am}$ and evening $5 \mathrm{pm}$ to $6 \mathrm{pm}$ during research period from June 2013 to May 2015. A liter of water sample from sampling stations was collected for the qualitative and quantitative estimation of phytoplankton study. Samples were subjected to use for further investigation by standard methods. Phytoplankton were identified according to Fritsch (1975), and standard Manuals and published papers, Sreenivasa et.al. (1973), Santhanam et.al. (1987) and Tomas (1995).

The water samples collected from the lake in bottles were brought to the laboratory for analysis as per the standard methods described by APHA (1985) and Trivedy and Goel(1986). Four sampling sites were identified almost equidistant on the shore of the reservoir to its north, east, west and south. After that the overlying water from the bottle was decanted and the final volume was adjusted in between 10 to $15 \mathrm{~m} 1$. The latter method was usually used to compare whether there was any demerit of using the other method. No significant difference was observed. After collection the phytoplankton material was transferred to glass preserved permanently in Transeau's solution (Distilled water $60 \mathrm{~m} 1$ or 6 parts; Absolute alcohol $30 \mathrm{~m} 1$ or 3 parts; Formaldehyde 10 $\mathrm{ml}$ or 1 part. To each $100 \mathrm{ml}$ of the above solution $5 \mathrm{~m} 1$ of glycerine was added to prevent the materials from becoming brittle (Transeau, 1951).

\section{Results and Discussion}

An extensive study was made to find out the occurrence and abundance of algae population in different study sites (FOUR) of Kamalapur, Karimnagar District, Telangana, India. Totally 56 species of algae belonging to genera fewer than 4 Classes viz., were recorded during the study period as shown in Table: no.(2). The distribution of these algal forms might be indicating the lower nitrogen status in all studied areas. Present study documented a remarkable biodiversity in species composition. Was the dominant genus of Cyanophyceae of studied area, Blue green algae are one of the major components of the nitrogen fixing biomass in ponds. Finally, it might be concluded that the documentation on Cyanobacteria may enhance the understanding.

The results of study area in different aspects like species composition Bacillariophyceae, Chlorophyceae, Cyanophyceae, Euglenophyceae i.e.4, 31,17,4.Generic composition 4,22,14,3.species wise and class wise percentage are Chlorophyceae I,93.5,II 77.41,III,100, IV,79.99. Bacillariophyceae I, 25, II, 75, 100, IV 75. Cyanophyceae, I94.11, II, 64.70, III, 88.25 IV, 82.35. Euglenophyceae I100, II, 100, III 50, IV 50. Species dominance based on their appearance from collected areas, Bacillariophyceae in Site I, 0, II, 25, III25, IV 0. Chlorophyceae I, 16.12 II, 32.25 III, 35.48 IV, 16.12. Cyanophyceae I, 29.41 II, 11.76 III, 11.76 IV, 5.6. Euglenophyceae I, $25 \%$ only.

Macrophytes of study area are 91 species belonging to Monocots, Dicots, Pterridophytes. Total genera are 68. Table no. (1) Macrophytes classification varies from literature one author to another. In present study we classify them as (EA) emergent anchored, (FF) free floating, 
(RFL) rooted and floating, (SA) submerged and anchored. Table No. (4). Family and species wise results are shown in Table No. (3).

Community production and respiration vary with season (Kemp et al. 1992; Smith and Hollibaugh 1997), salinity (Swaney et al. 1999) and depth (Caffrey et al. 1998). Gomes et al (2000) observed increased phytoplankton biomass (92 mg m-2) during monsoon with reference to increased river runoff and wind driven coastal upwelling on the west coast of Bay of Bengal compared to other seasons, however, the primary productivity was low, suggesting light limitation due to intense cloud cover and high suspended load associated with higher river discharge. Phytoplankton appears to have rapidly consumed nutrients during post monsoon bloom. However, near saturation levels, coinciding with short-term blooms in dry periods and in October indicate dominant photosynthetic activity over respiration. Importantly, though pigments of photosynthesis content in OctoberNovember bloom was double to that found during the January-February bloom, the oxygen was clearly under saturated during the former period.

Table.1 Enumeration of Macrophytes of Kamalapur

\begin{tabular}{|c|c|c|c|c|c|c|c|c|c|}
\hline \multirow[t]{2}{*}{ Sl no } & \multirow[t]{2}{*}{ Scientific name } & \multirow[t]{2}{*}{ Family } & \multirow[t]{2}{*}{ Habit } & \multicolumn{4}{|c|}{ SITES } & \multirow[t]{2}{*}{$\begin{array}{c}\text { Life } \\
\text { form }\end{array}$} & \multirow{2}{*}{\begin{tabular}{|c} 
IUCN \\
Version \\
2015.3 \\
\end{tabular}} \\
\hline & & & & I & II & III & IV & & \\
\hline 1 & Aerva laneta & Amaranthaceae & $\mathrm{H}$ & $\mathrm{P}$ & $\mathrm{A}$ & $\mathrm{A}$ & $\mathrm{P}$ & EA & $\mathrm{NE}$ \\
\hline 2 & Aeschynomene aspera L. & Fabaceae & Us & $\mathrm{P}$ & $\mathrm{P}$ & $\mathrm{A}$ & $\mathrm{P}$ & EA & $\mathrm{LC}$ \\
\hline 3 & A.diffusa Wild & Fabaceae & Us & $\mathrm{A}$ & $\mathrm{A}$ & $\mathrm{P}$ & $\mathrm{P}$ & EA & $\mathrm{NE}$ \\
\hline 4 & Ageratum conyzoides L. & Asteraceae & $\mathrm{H}$ & $\mathrm{P}$ & $\mathrm{P}$ & $\mathrm{P}$ & $\mathrm{P}$ & EA & $\mathrm{NE}$ \\
\hline 5 & Alysicarpus rugosus & Fabaceae & $\mathrm{Cr}$ & $\mathrm{P}$ & $\mathrm{P}$ & $\mathrm{P}$ & $\mathrm{P}$ & EA & $\mathrm{NE}$ \\
\hline 6 & A. vaginalis (L) DC & Fabaceae & $\mathrm{Cr}$ & $\mathrm{P}$ & A & $\mathrm{P}$ & $\mathrm{P}$ & EA & $\mathrm{NE}$ \\
\hline 7 & Ammania buccifera & Lythraceae & $\mathrm{H}$ & $\mathrm{P}$ & $\mathrm{P}$ & $\mathrm{P}$ & $\mathrm{P}$ & EA & $\mathrm{NE}$ \\
\hline 8 & A.roxiburghi & Lythraceae & $\mathrm{H}$ & $\mathrm{P}$ & $\mathrm{P}$ & $\mathrm{P}$ & $\mathrm{P}$ & EA & $\mathrm{NE}$ \\
\hline 9 & $\begin{array}{l}\text { Alternanthera } \\
\text { philoxeroides (Mar) Grisep. }\end{array}$ & Amaranthaceae & $\mathrm{Cr}$ & $\mathrm{P}$ & $\mathrm{P}$ & $\mathrm{P}$ & $\mathrm{P}$ & EA & $\mathrm{NE}$ \\
\hline 10 & A. sessilis (L.) R.Br.ex DC. & Amaranthaceae & $\mathrm{Cr}$ & $\mathrm{P}$ & $\mathrm{P}$ & $\mathrm{P}$ & $\mathrm{P}$ & $\mathrm{SM}$ & DD \\
\hline 11 & Aponogeton natans & Aponogetonaceae & $\mathrm{H}$ & $\mathrm{P}$ & $\mathrm{A}$ & $\mathrm{P}$ & $\mathrm{A}$ & $\mathrm{SA}$ & $\mathrm{NE}$ \\
\hline 12 & Argemone mexicana $\mathrm{L}$. & Papaveraceae & $\mathrm{H}$ & $\mathrm{P}$ & $\mathrm{A}$ & $\mathrm{P}$ & $\mathrm{P}$ & EA & $\mathrm{NE}$ \\
\hline 13 & Aurundo donax L. & Poaceae & $\mathrm{H}$ & $\mathrm{P}$ & $\mathrm{P}$ & $\mathrm{P}$ & $\mathrm{P}$ & EA & $\mathrm{NE}$ \\
\hline 14 & Azolla pinnata $R$. Br. & Salviniceae & $\mathrm{H}$ & $\mathrm{P}$ & $\mathrm{P}$ & $\mathrm{P}$ & $\mathrm{A}$ & $\mathrm{FF}$ & $\mathrm{LC}$ \\
\hline 15 & Bergia capensis & Elatinaceae & $\mathrm{H}$ & $\mathrm{P}$ & $\mathrm{A}$ & $\mathrm{P}$ & $\mathrm{A}$ & EA & $\mathrm{NE}$ \\
\hline 16 & Blumea axillaris & Asteraceae & $\mathrm{H}$ & $\mathrm{P}$ & $\mathrm{A}$ & $\mathrm{P}$ & $\mathrm{A}$ & EA & $\mathrm{NE}$ \\
\hline 17 & Breniya retusa & Euphorbiaceae & $\mathrm{H}$ & $\mathrm{P}$ & $\mathrm{P}$ & $\mathrm{P}$ & A & EA & $\mathrm{NE}$ \\
\hline 18 & Ceratophyllum demersum L. & Ceratophyllaceae & $\mathrm{H}$ & $\mathrm{P}$ & $\mathrm{P}$ & $\mathrm{P}$ & $\mathrm{P}$ & SA & $\mathrm{NE}$ \\
\hline 19 & Chrozophora rottleri & Euphorbiaceae & $\mathrm{H}$ & $\mathrm{P}$ & A & $\mathrm{P}$ & $\mathrm{P}$ & EA & $\mathrm{LC}$ \\
\hline 20 & Cleome chelidoni & Capparaceae & $\mathrm{H}$ & $\mathrm{P}$ & $\mathrm{A}$ & $\mathrm{P}$ & $\mathrm{P}$ & EA & $\mathrm{NE}$ \\
\hline 21 & Coldenia procumbena & Boraginaceae & $\mathrm{Cr}$ & $\mathrm{P}$ & $\mathrm{P}$ & $\mathrm{P}$ & $\mathrm{P}$ & EA & $\mathrm{NE}$ \\
\hline 22 & Commelina benghalensis L. & Commelinaceae & $\mathrm{H}$ & $\mathrm{P}$ & $\mathrm{P}$ & $\mathrm{P}$ & $\mathrm{P}$ & SA & $\mathrm{LC}$ \\
\hline 23 & C. haskarhi. $\mathbf{L}$ & Commelinaceae & $\mathrm{H}$ & $\mathrm{P}$ & $\mathrm{P}$ & $\mathrm{P}$ & $\mathrm{P}$ & $\mathrm{SA}$ & $\mathrm{NE}$ \\
\hline
\end{tabular}




\begin{tabular}{|c|c|c|c|c|c|c|c|c|c|}
\hline 24 & Croton banaplandium & Euphorbiaceae & $\mathrm{H}$ & $\mathrm{P}$ & A & $\mathrm{P}$ & $\mathrm{P}$ & EA & $\mathrm{NE}$ \\
\hline 25 & Cyanotis bonblandianum & Commelinaceae & $\mathrm{H}$ & $\mathrm{P}$ & A & $\mathrm{P}$ & $\mathrm{P}$ & SA & LC \\
\hline 26 & Cynodon dactylon (L) Pers. & Poaceae & $\mathrm{H}$ & $\mathrm{P}$ & $\mathrm{P}$ & $\mathrm{P}$ & $\mathrm{P}$ & EA & $\mathrm{NE}$ \\
\hline 27 & Cyperus arenarius Retz & Cyperaceae & $\mathrm{H}$ & $\mathrm{P}$ & $\mathrm{P}$ & $\mathrm{P}$ & $\mathrm{P}$ & SA & $\mathrm{LC}$ \\
\hline 28 & C. compressus $\mathrm{L}$. & Cyperaceae & $\mathrm{H}$ & $\mathrm{P}$ & $\bar{A}$ & $\mathrm{P}$ & $\mathrm{P}$ & EA & $\mathrm{LC}$ \\
\hline 29 & C. corymbosus Rottb. & Cyperaceae & $\mathrm{H}$ & $\mathrm{P}$ & A & $\mathrm{P}$ & $\mathrm{P}$ & EA & $\mathrm{LC}$ \\
\hline 30 & C.difformis & Cyperaceae & $\mathrm{H}$ & $\mathrm{P}$ & $\mathrm{P}$ & $\mathrm{P}$ & $\mathrm{P}$ & EA & $\mathrm{LC}$ \\
\hline 31 & C.eragrostis & Cyperaceae & $\mathrm{H}$ & $\mathrm{P}$ & $\mathrm{P}$ & $\mathrm{P}$ & $\mathrm{P}$ & EA & LC \\
\hline 32 & C.exaltanus Retz & Cyperaceae & $\mathrm{H}$ & $\mathrm{A}$ & $\mathrm{P}$ & A & $\mathrm{P}$ & EA & LC \\
\hline 33 & C. rotundus $\mathrm{L}$ & Cyperaceae & $\mathrm{H}$ & $\mathrm{P}$ & A & $\mathrm{P}$ & $\mathrm{A}$ & EA & LC \\
\hline 34 & C.tenuispica Steud & Cyperaceae & $\mathrm{H}$ & $\mathrm{A}$ & $\mathrm{P}$ & A & $\mathrm{P}$ & EA & LC \\
\hline 35 & Dentella repens Forst. & Rubiaceae & $\mathrm{H}$ & $\mathrm{P}$ & A & $\mathrm{P}$ & $\mathrm{P}$ & EA & $\mathrm{LC}$ \\
\hline 36 & $\begin{array}{l}\text { Desmodium triflorum (L.) } \\
\text { DC. }\end{array}$ & Fabaceae & $\mathrm{Cr}$ & $\mathrm{P}$ & $\mathrm{P}$ & $\mathrm{P}$ & $\mathrm{P}$ & EA & $\mathrm{LC}$ \\
\hline 37 & Digitaria sanguinalis & Poaceae & $\mathrm{H}$ & $\mathrm{P}$ & $\mathrm{P}$ & $\mathrm{P}$ & $\mathrm{P}$ & EA & $\mathrm{NE}$ \\
\hline 38 & Eclipta alba. (L.) L. & Asteraceae & $\mathrm{H}$ & $\mathrm{P}$ & $\mathrm{P}$ & $\mathrm{P}$ & $\mathrm{P}$ & EA & $\mathrm{DD}$ \\
\hline 39 & E. prostrata (L.) L. & Asteraceae & $\mathrm{H}$ & $\mathrm{P}$ & $\mathrm{P}$ & $\mathrm{P}$ & $\mathrm{P}$ & EA & $\mathrm{DD}$ \\
\hline 40 & $\begin{array}{l}\text { Eichhornia crassipes (Mart.) } \\
\text { S.L. }\end{array}$ & Pontederiaceae & $\mathrm{H}$ & $\mathrm{P}$ & A & A & $\mathrm{P}$ & FF & $\mathrm{NE}$ \\
\hline 41 & $\begin{array}{|ll|}\text { Fimbristylis } & \text { argentia } \\
\text { (Rottb.)Vahl }\end{array}$ & Cyperaceae & $\mathrm{H}$ & $\mathrm{P}$ & $\mathrm{P}$ & $\mathrm{P}$ & A & EA & $\mathrm{LC}$ \\
\hline 42 & F.dichotoma $(L)$ Vahl & Cyperaceae & $\mathrm{H}$ & $\mathrm{A}$ & $\mathrm{P}$ & $\mathrm{A}$ & $\mathrm{P}$ & EA & $\mathrm{LC}$ \\
\hline 43 & F.miliacea & Cyperaceae & $\mathrm{H}$ & $\mathrm{A}$ & $\mathrm{P}$ & $\mathrm{A}$ & $\mathrm{P}$ & EA & $\mathrm{LC}$ \\
\hline 44 & F.triflora & Cyperaceae & $\mathrm{H}$ & $\mathrm{A}$ & $\mathrm{P}$ & $\mathrm{P}$ & $\mathrm{P}$ & EA & $\mathrm{LC}$ \\
\hline 45 & Glinnus lotoides & Molluginaceae & $\mathrm{Cr}$ & $\mathrm{P}$ & $\mathrm{P}$ & $\mathrm{P}$ & $\mathrm{P}$ & EA & $\mathrm{NE}$ \\
\hline 46 & G.oppositifolia & Molluginaceae & $\mathrm{Cr}$ & $\mathrm{P}$ & A & $\mathrm{P}$ & $\mathrm{P}$ & & \\
\hline 47 & $\begin{array}{l}\text { Grangea maderaspatana (L.) } \\
\text { Poir. }\end{array}$ & Asteraceae & $\mathrm{H}$ & A & $\mathrm{P}$ & A & $\mathrm{P}$ & SA & $\mathrm{LC}$ \\
\hline 48 & Heliotrophium curasavica & Boraginaceae & $\mathrm{H}$ & $\mathrm{P}$ & $\mathrm{P}$ & $\mathrm{P}$ & $\mathrm{P}$ & EA & $\mathrm{NE}$ \\
\hline 49 & H.indica & Boraginaceae & $\mathrm{H}$ & $\mathrm{P}$ & $\mathrm{P}$ & $\mathrm{P}$ & $\mathrm{P}$ & EA & $\mathrm{NE}$ \\
\hline 50 & H.zeylanica & Bor: & $\mathrm{H}$ & $\mathrm{P}$ & A & $\mathrm{P}$ & $\mathrm{P}$ & EA & $\mathrm{NE}$ \\
\hline 51 & \begin{tabular}{|l}
$\begin{array}{l}\text { Hydrilla } \\
\text { Royle. }\end{array}$ \\
Rerticillata (L.f.) \\
\end{tabular} & Hydrocharitaceae & $\mathrm{H}$ & $\mathrm{P}$ & $\mathrm{P}$ & $\mathrm{P}$ & $\mathrm{P}$ & SA & LC \\
\hline 52 & Hygrophila sculli. & Acanthaceae & $\mathrm{H}$ & $\mathrm{P}$ & A & $\mathrm{P}$ & $\mathrm{A}$ & SA & $\mathrm{NE}$ \\
\hline 53 & $\begin{array}{l}\begin{array}{l}\text { Hygroryza aristata (Retz.) } \\
\text { Nees. }\end{array} \\
\end{array}$ & Poaceae & $\mathrm{H}$ & $\mathrm{P}$ & $\mathrm{P}$ & $\mathrm{P}$ & $\mathrm{P}$ & RFL & $\mathrm{NE}$ \\
\hline 54 & Indigofera linnaie & Fabaceae & $\mathrm{Cr}$ & $\mathrm{A}$ & $\mathrm{P}$ & $\mathrm{P}$ & $\mathrm{P}$ & EA & $\mathrm{NE}$ \\
\hline 55 & I. trita & Fabaceae & $\mathrm{Cr}$ & $\mathrm{P}$ & A & A & $\mathrm{P}$ & EA & $\mathrm{NE}$ \\
\hline 56 & Ipomoea aquatica Forssk. & Convolvulaceae & $\mathrm{H}$ & $\mathrm{P}$ & $\mathrm{P}$ & $\mathrm{P}$ & $\mathrm{P}$ & RFL & $\mathrm{LC}$ \\
\hline 57 & I. carnea Jaeq. & Convolvulaceae & $\mathrm{Sh}$ & $\mathrm{P}$ & $\mathrm{P}$ & $\mathrm{P}$ & $\mathrm{P}$ & EA & $\mathrm{NE}$ \\
\hline 58 & Kyllinga monocephela Roxb. & Cyperaceae & $\mathrm{H}$ & $\mathrm{P}$ & $\mathrm{P}$ & $\mathrm{P}$ & $\mathrm{P}$ & EA & $\mathrm{NE}$ \\
\hline 59 & Leersia hexandra $\mathrm{Sw}$. & Poaceae & $\mathrm{H}$ & $\mathrm{P}$ & A & $\mathrm{P}$ & $\mathrm{P}$ & EA & $\mathrm{NE}$ \\
\hline 60 & Lemna purpusilla Torrey & Lemnace & $\mathrm{H}$ & $\mathrm{P}$ & $\mathrm{P}$ & $\mathrm{P}$ & $\mathrm{P}$ & FF & $\mathrm{NE}$ \\
\hline 61 & Leucas aspera Link & Lamiaceae & $\mathrm{H}$ & $\mathrm{P}$ & A & $\mathrm{P}$ & $\mathrm{A}$ & EA & $\mathrm{NE}$ \\
\hline
\end{tabular}




\begin{tabular}{|c|c|c|c|c|c|c|c|c|c|}
\hline 62 & \begin{tabular}{|l} 
Ludwigia adscandens (L.) \\
Hara
\end{tabular} & Onagraceae & $\mathrm{H}$ & $\mathrm{P}$ & $\mathrm{P}$ & $\mathrm{P}$ & $\mathrm{P}$ & RFL & $\mathrm{NE}$ \\
\hline 63 & L. parviflora Roxb. & Onagraceae & $\mathrm{H}$ & $\mathrm{P}$ & A & $\mathrm{P}$ & A & EA & $\mathrm{NE}$ \\
\hline 64 & L. perennis $\mathrm{L}$. & Onagraceae & $\mathrm{H}$ & $\mathrm{A}$ & A & $\mathrm{P}$ & $\mathrm{P}$ & EA & $\mathrm{NE}$ \\
\hline 65 & Marsalia quadrifolia $L$. & Marseliaceae & $\mathrm{H}$ & $\mathrm{P}$ & $\mathrm{P}$ & $\mathrm{P}$ & $\mathrm{P}$ & EA & $\mathrm{NE}$ \\
\hline 66 & Merrimia emariginum & Convolvulaceae & $\mathrm{Cr}$ & $\mathrm{P}$ & $\mathrm{A}$ & $\mathrm{P}$ & $\mathrm{A}$ & EA & $\mathrm{NE}$ \\
\hline 67 & Murdania nudiflora & Commelinaceae & $\mathrm{H}$ & $\mathrm{P}$ & $\mathrm{P}$ & $\mathrm{P}$ & $\mathrm{A}$ & SA & $\mathrm{NE}$ \\
\hline 68 & Najas indica (Willd.) Cham. & Najadaceae & $\mathrm{H}$ & $\mathrm{P}$ & $\mathrm{P}$ & $\mathrm{P}$ & $\mathrm{P}$ & SA & $\mathrm{LC}$ \\
\hline 69 & N.minor & Najadaceae & $\mathrm{H}$ & $\mathrm{P}$ & A & $\mathrm{P}$ & $\mathrm{P}$ & SA & $\mathrm{LC}$ \\
\hline 70 & Nymphaea alba $\mathrm{L}$. & Nymphaeaceae & $\mathrm{H}$ & $\mathrm{P}$ & $\mathrm{P}$ & $\mathrm{P}$ & A & RFL & $\mathrm{LC}$ \\
\hline 71 & N.nouchali Burm.f. & Nymphaeaceae & $\mathrm{H}$ & $\mathrm{P}$ & $\mathrm{P}$ & $\mathrm{P}$ & $\mathrm{P}$ & RFL & $\mathrm{LC}$ \\
\hline 72 & $\begin{array}{l}\text { Nymphoides } \\
\text { cristata (Roxb.)Kuntze }\end{array}$ & Nymphaeaceae & $\mathrm{H}$ & $\mathrm{P}$ & $\mathrm{P}$ & $\mathrm{P}$ & $\mathrm{P}$ & RFL & $\mathrm{LC}$ \\
\hline 73 & N.indica (L.) Kuntze & Nymphaeaceae & $\mathrm{H}$ & $\mathrm{P}$ & $\mathrm{P}$ & $\mathrm{P}$ & $\mathrm{P}$ & RFL & $\mathrm{LC}$ \\
\hline 74 & Ottelia alismoides (L.) Pers. & Hydrocharitaceae & $\mathrm{H}$ & $\mathrm{P}$ & $\mathrm{P}$ & $\mathrm{P}$ & $\mathrm{P}$ & SA & $\mathrm{LC}$ \\
\hline 75 & Phalaris aurundinosa & Poaceae & $\mathrm{H}$ & $\mathrm{P}$ & $\mathrm{A}$ & $\mathrm{P}$ & $\mathrm{A}$ & SA & $\mathrm{NE}$ \\
\hline 76 & Phyla nodiflora (L.) Greene & Verbenaceae & $\mathrm{H}$ & $\mathrm{P}$ & $\mathrm{P}$ & $\mathrm{P}$ & $\mathrm{P}$ & SA & $\mathrm{LC}$ \\
\hline 77 & Oxlis corniculata $\mathrm{L}$. & Oxalidaceae & $\mathrm{H}$ & $\mathrm{P}$ & $\mathrm{A}$ & $\mathrm{P}$ & $\mathrm{P}$ & SA & $\mathrm{NE}$ \\
\hline 78 & Panicum repens $\mathrm{L}$. & Poaceae & $\mathrm{H}$ & $\mathrm{P}$ & A & $\mathrm{P}$ & A & SA & $\mathrm{LC}$ \\
\hline 79 & Parthenium hysterophorus L. & Asteraceae & $\mathrm{H}$ & $\mathrm{P}$ & $\mathrm{P}$ & $\mathrm{P}$ & $\mathrm{P}$ & EA & $\mathrm{NE}$ \\
\hline 80 & Pistia stratiotes $\mathrm{L}$. & Araceae & $\mathrm{H}$ & $\mathrm{P}$ & $\mathrm{P}$ & A & A & FF & $\mathrm{NE}$ \\
\hline 81 & Rorippa palustris & Brassicaceae & $\mathrm{H}$ & $\mathrm{P}$ & $\mathrm{P}$ & $\mathrm{P}$ & $\mathrm{P}$ & EA & $\mathrm{NE}$ \\
\hline 82 & Polygonum plebiumL. & Polygonaceae & $\mathrm{H}$ & $\mathrm{P}$ & A & $\mathrm{P}$ & $\mathrm{P}$ & EA & $\mathrm{NE}$ \\
\hline 83 & Potomogeton crispus L. & Potamogetonaceae & $\mathrm{H}$ & $\mathrm{P}$ & $\mathrm{A}$ & $\mathrm{P}$ & $\mathrm{P}$ & SA & $\mathrm{NE}$ \\
\hline 84 & P.pectinatus & Potamogetonaceae & $\mathrm{H}$ & $\mathrm{P}$ & $\mathrm{P}$ & $\mathrm{P}$ & A & SA & $\mathrm{NE}$ \\
\hline 85 & Scirpus articulatus L. & Cyperaceae & $\mathrm{H}$ & $\mathrm{P}$ & $\mathrm{P}$ & $\mathrm{P}$ & $\mathrm{P}$ & EA & $\mathrm{NE}$ \\
\hline 86 & Spheranthus indicus & Asteraceae & $\mathrm{Cr}$ & $\mathrm{P}$ & A & $\mathrm{P}$ & A & EA & $\mathrm{NE}$ \\
\hline 87 & Trianthema portulacanthem & Portulacaeae & $\mathrm{H}$ & $\mathrm{P}$ & $\mathrm{P}$ & $\mathrm{P}$ & $\mathrm{P}$ & EA & $\mathrm{NE}$ \\
\hline 88 & Typhaa aungstifolia. & Typhaceae & $\mathrm{H}$ & $\mathrm{P}$ & $\mathrm{P}$ & $\mathrm{P}$ & A & EA & $\mathrm{NE}$ \\
\hline 89 & Urena lobata $\mathrm{L}$. & Malvaceae & $\mathrm{H}$ & $\mathrm{P}$ & A & $\mathrm{P}$ & A & EA & $\mathrm{NE}$ \\
\hline 90 & Valisnaria spiralis Linn. & Hydrocharitaceae & $\mathrm{H}$ & $\mathrm{P}$ & $\mathrm{P}$ & $\mathrm{P}$ & $\mathrm{P}$ & SA & $\mathrm{NE}$ \\
\hline 91 & Xanthium indicm & Asteraceae & $\mathrm{H}$ & $\mathrm{P}$ & $\mathrm{P}$ & $\mathrm{P}$ & $\mathrm{P}$ & EA & $\mathrm{NE}$ \\
\hline Total & & & 91 & 84 & 60 & 81 & 71 & & \\
\hline
\end{tabular}

$\mathrm{H}=$ herb, $\mathrm{Cr}=$ creeper,Sh=shrub,Us=under shrub. $\mathrm{P}=$ present, $\mathrm{A}=$ absent,

$\mathrm{EA}=$ emergent anchored, $\mathrm{FF}=$ free floating, $\mathrm{RFL}=$ rooted and floating, $\mathrm{SA}=$ submerged and anchored.

$\mathrm{NE}=$ not evaluated, $\mathrm{LC}=$ least concern, $\mathrm{DD}=$ data deficient 
Table.2 Phytoplnkton Distribution

\begin{tabular}{|c|c|c|c|c|c|c|c|}
\hline S.No & Algal species & Class & $\begin{array}{c}\text { Site } \\
\text { I }\end{array}$ & $\begin{array}{l}\text { Site } \\
\text { II }\end{array}$ & $\begin{array}{l}\text { Site } \\
\text { III }\end{array}$ & $\begin{array}{l}\text { Site } \\
\text { IV }\end{array}$ & Total \\
\hline 1 & $\begin{array}{l}\text { Fragillaria brevistriata } \\
\text { Grun }\end{array}$ & Bacillariophyceae & - & $*$ & $*$ & - & 2 \\
\hline 2 & $\begin{array}{l}\text { Navicula cuspidate } \\
\text { Kuetz. }\end{array}$ & Bacillariophyceae & - & $*$ & $*$ & $*$ & 3 \\
\hline 3 & Pinnularia gibba Ehr. & Bacillariophyceae & - & - & $*$ & $*$ & 2 \\
\hline 4 & Synedra ulna (Nitz) & Bacillariophyceae & $*$ & $* *$ & $* *$ & $*$ & 4 \\
\hline 5 & $\begin{array}{l}\text { Ankistrodesmus } \\
\text { falcatus (Corda) Ralfs }\end{array}$ & Chlorophyceae & $*$ & $*$ & $*$ & $*$ & 4 \\
\hline 6 & Anthodesmus curuvatus & Chlorophyceae & - & - & $*$ & $*$ & 2 \\
\hline 7 & Chara vulgaris & Chlorophyceae & $*$ & $*$ & $*$ & $*$ & 4 \\
\hline 8 & $\begin{array}{l}\text { Chlamydomonas } \\
\text { globosa Snow. }\end{array}$ & Chlorophyceae & $*$ & $*$ & $*$ & - & 2 \\
\hline 9 & $\begin{array}{l}\text { Chlorella ellipsoidea } \\
\text { Gerneck }\end{array}$ & Chlorophyceae & - & - & $*$ & - & $\mathbf{1}$ \\
\hline 10 & C. vulgaris Beyernick & Chlorophyceae & $*$ & - & $*$ & - & 2 \\
\hline 11 & $\begin{array}{l}\text { Closterium acerosum } \\
\text { (Schrank) Ehr. }\end{array}$ & Chlorophyceae & $*$ & $*$ & $*$ & $*$ & 3 \\
\hline 12 & C.tumidum & Chlorophyceae & $*$ & $*$ & $*$ & - & 2 \\
\hline 13 & Cladophora glomerata & Chlorophyceae & $*$ & $*$ & $*$ & $*$ & 3 \\
\hline 14 & $\begin{array}{l}\text { Cosmarium botrytis } \\
\text { Menegh }\end{array}$ & Chlorophyceae & $*$ & $* *$ & $* *$ & $*$ & 4 \\
\hline 15 & C.auriculata & Chlorophyceae & $*$ & $*$ & $*$ & $*$ & 4 \\
\hline 16 & C.granatum & Chlorophyceae & $*$ & - & $*$ & - & 2 \\
\hline 17 & Microspora sp & Chlorophyceae & $*$ & $*$ & $*$ & - & 3 \\
\hline 18 & Nitella $s p$ & Chlorophyceae & $*$ & $* *$ & $* *$ & $*$ & 4 \\
\hline 19 & $\begin{array}{l}\text { Oedogonium } \\
\text { borisianum }\end{array}$ & Chlorophyceae & $* *$ & $* *$ & $* *$ & $* *$ & 4 \\
\hline 20 & $O . s p$ & Chlorophyceae & $*$ & $* *$ & $*$ & $*$ & 4 \\
\hline 21 & Oocystis gigas & Chlorophyceae & $* *$ & $*$ & $* *$ & $* *$ & 4 \\
\hline 22 & Pandorina morum Bory & Chlorophyceae & $*$ & $* *$ & $*$ & $*$ & 4 \\
\hline 23 & $\begin{array}{l}\text { Pediastrum biradiatum } \\
\text { Presc }\end{array}$ & Chlorophyceae & $*$ & $*$ & $*$ & $*$ & 3 \\
\hline 24 & P.duplex & Chlorophyceae & $*$ & $* *$ & $* *$ & $*$ & 4 \\
\hline 25 & P.simplex & Chlorophyceae & $*$ & $*$ & $* *$ & $*$ & 4 \\
\hline 26 & Pithophora varia & Chlorophyceae & $*$ & $* *$ & $* *$ & $* *$ & 4 \\
\hline 27 & $\begin{array}{l}\text { Rhizoclonium } \\
\text { hieroglypicum }\end{array}$ & Chlorophyceae & $*$ & $*$ & $*$ & $*$ & 4 \\
\hline 28 & $\begin{array}{l}\text { Scenidesmus } \\
\text { dendiculatum }\end{array}$ & Chlorophyceae & $*$ & $* *$ & $* *$ & $* *$ & 4 \\
\hline 29 & S.quadracauda & Chlorophyceae & $*$ & $* *$ & $* *$ & $*$ & 4 \\
\hline 30 & S.dimorphosus & Chlorophyceae & $* *$ & - & $*$ & $*$ & 3 \\
\hline
\end{tabular}




\begin{tabular}{|c|c|c|c|c|c|c|c|}
\hline 31 & Spirogyra formosa & Chlorophyceae & $* *$ & $* *$ & $* *$ & $*$ & 4 \\
\hline 32 & Staurastrum pinnatum & Chlorophyceae & $*$ & - & $*$ & $*$ & 3 \\
\hline 33 & Tetraedron quadratum & Chlorophyceae & $*$ & $*$ & $*$ & $*$ & 4 \\
\hline 34 & Ulothrix sp & Chlorophyceae & $*$ & - & $*$ & $*$ & 3 \\
\hline 35 & Zygnema czurde & Chlorophyceae & $* *$ & $*$ & $* *$ & $* *$ & 4 \\
\hline 36 & Anabaena constricta & Cyanophyceae & $*$ & $*$ & $* *$ & $*$ & 4 \\
\hline 37 & A. iyengarii & Cyanophyceae & * & * & * & $*$ & 4 \\
\hline 38 & Anabaenopsis sp & Cyanophyceae & $* *$ & $*$ & $*$ & $* *$ & 4 \\
\hline 39 & Apanocapsa litorates & Cyanophyceae & $*$ & $*$ & $*$ & - & 3 \\
\hline 40 & $\begin{array}{l}\text { Arthrospira platensis } \\
\text { (Nordst }\end{array}$ & Cyanophyceae & * & - & $*$ & * & 3 \\
\hline 41 & Chroococus dispenses & Cyanophyceae & $*$ & $*$ & $*$ & $*$ & 4 \\
\hline 42 & C.turgidus & Cyanophyceae & $*$ & - & $*$ & - & 2 \\
\hline 43 & Coeolophaeriumdubium & Cyanophyceae & $*$ & - & $*$ & $*$ & 2 \\
\hline 44 & Gleocapsa sp. & Cyanophyceae & $*$ & $*$ & - & $*$ & 3 \\
\hline 45 & Microcystis erusinosa & Cyanophyceae & $* *$ & - & - & $*$ & 2 \\
\hline 46 & Nostoc commune & Cyanophyceae & $* *$ & $*$ & $* *$ & $*$ & 4 \\
\hline 47 & Oscillatoria tenuis & Cyanophyceae & $* *$ & $*$ & $*$ & $*$ & 4 \\
\hline 48 & O.rubesenes & Cyanophyceae & - & - & $*$ & $*$ & 2 \\
\hline 49 & Lyngbya ceylanica & Cyanophyceae & $*$ & $* *$ & $* *$ & $*$ & 4 \\
\hline 50 & Phormidium tennue & Cyanophyceae & $*$ & $*$ & $*$ & - & 3 \\
\hline 51 & $\begin{array}{l}\text { Spirulina major (Kütz) } \\
\text { Gomont }\end{array}$ & Cyanophyceae & $*$ & - & $*$ & $*$ & 3 \\
\hline 52 & Synechococcus aponina & Cyanophyceae & $* *$ & $* *$ & $*$ & $*$ & 4 \\
\hline 53 & Euglena cadata & Euglenophyceae & $*$ & $*$ & - & $*$ & 3 \\
\hline 54 & Phacus acuminatus & Euglenophyceae & $*$ & $*$ & $*$ & - & 3 \\
\hline 55 & P.longicauda & Euglenophyceae & $*$ & $*$ & $*$ & - & 3 \\
\hline 56 & Lepocinclis fusiformis & Euglenophyceae & $* *$ & $*$ & - & $*$ & 3 \\
\hline \multicolumn{3}{|c|}{ Total } & 50 & 42 & 52 & 44 & \\
\hline
\end{tabular}


Table.3 Families Wise and Species Wise

\begin{tabular}{|l|l|l|l|}
\hline S. No & Family & $\begin{array}{l}\text { Species } \\
\text { No. }\end{array}$ & Monospecies families \\
\hline 1 & Cyperaceae & 14 & Acanthaceae Araceae \\
\hline 2 & Asteraceae & 8 & Brassicaceae \\
\hline 3 & Fabaceae & 7 & $\begin{array}{l}\text { Capparaceae } \\
\text { Ceratophyllaceae }\end{array}$ \\
\hline 4 & Poaceae & 7 & Elatinaceae \\
\hline 5 & Boraginaceae & 4 & Lamiaceae, Lemnaceae \\
\hline 67 & Commilinaceae & 4 & $\begin{array}{l}\text { Malvaceae } \\
\text { Marseliaceae }\end{array}$ \\
\hline 8 & Nymphaceae & 4 & Oxalidaceae \\
\hline 9 & Amaranthaceae & 3 & $\begin{array}{l}\text { Papavaraceae } \\
\text { Polygonaceae }\end{array}$ \\
\hline 10 & Convolvulaceae & 3 & $\begin{array}{l}\text { Pontederiacea } \\
\text { Portulacaeae }\end{array}$ \\
\hline 11 & Euphorbiaceae & 3 & Rubiaceae \\
\hline 12 & Hydrocharitaceae & 3 & Salviniceae \\
\hline 13 & Onagraceae & 3 & Typhaceae \\
\hline 14 & Lythraceae & 2 & Verbenaceae \\
\hline 15 & Molluginaceae & 2 & \\
\hline 16 & Najadaceae & 2 & \\
\hline 17 & Potamogetanaceae & 2 & \\
\hline & & & \\
\hline
\end{tabular}

Table.4 Macrophytes Morphological, Life forms, IUCN Categories

\begin{tabular}{|l|l|l|l|l|}
\hline S.No & $\begin{array}{l}\text { Morphological } \\
\text { form }\end{array}$ & $\begin{array}{l}\text { IUCN } \\
\text { categories }\end{array}$ & $\begin{array}{l}\text { Life } \\
\text { form }\end{array}$ & $\begin{array}{l}\text { Site wise } \\
\text { distribution }\end{array}$ \\
\hline 1 & H-76 & LC-32 & EA-59 & I-84 \\
\hline 2 & Cr-12 & DD-04 & FF-04 & II-60 \\
\hline 3 & Us-02 & NE-55 & RFL-07 & III-81 \\
\hline 4 & Sh-01 & ---- & SA-21 & IV-71 \\
\hline
\end{tabular}

$\mathrm{H}=\mathrm{Herb}, \mathrm{Cr}=$ creeper, $\mathrm{Us}=$ under shrub, $\mathrm{Sh}=$ shrub.LC=least concern, $\mathrm{DD}=$ data deficient, $\mathrm{NE}=$ not evaluated. $\mathrm{EA}=$ emergent anchored, $\mathrm{FF}=$ free floating, $\mathrm{RFL}=$ rooted and floating, $\mathrm{SA}=$ submerged and anchored 


\section{Macrophytes: Plate No.1}

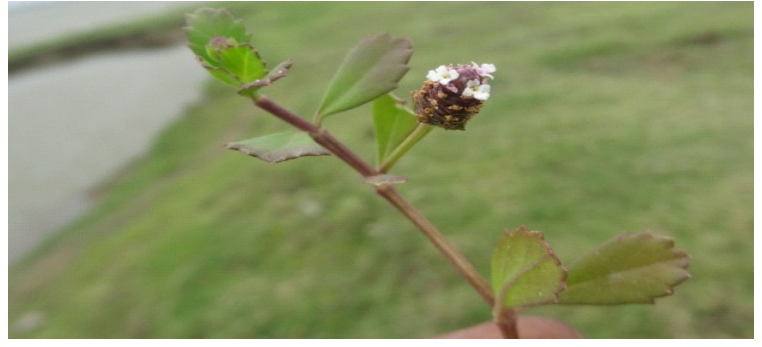

A) Phyla nodiflora (L.) Green e

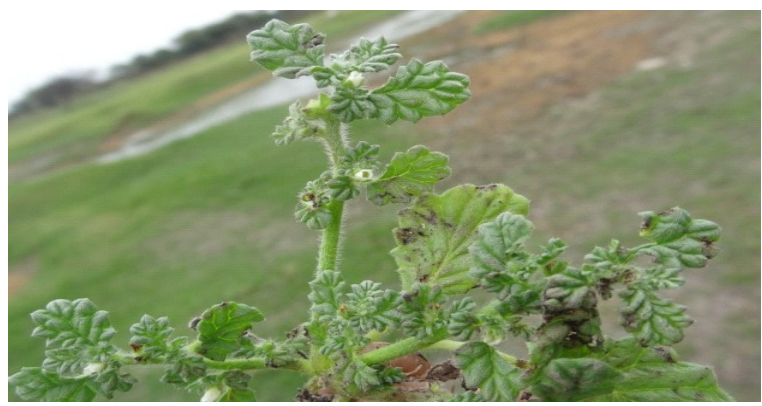

C) Coldenia procumbena

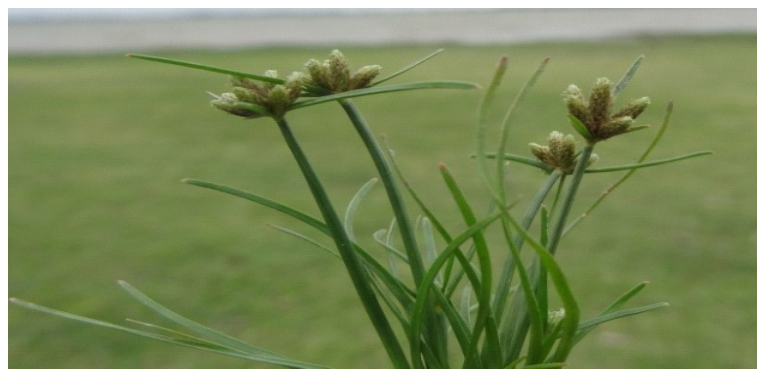

E) Fim bristylis argentia

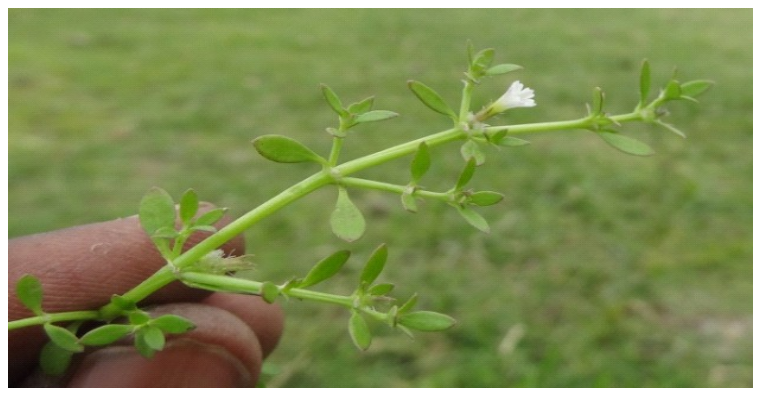

G ) Dentella repens Forst

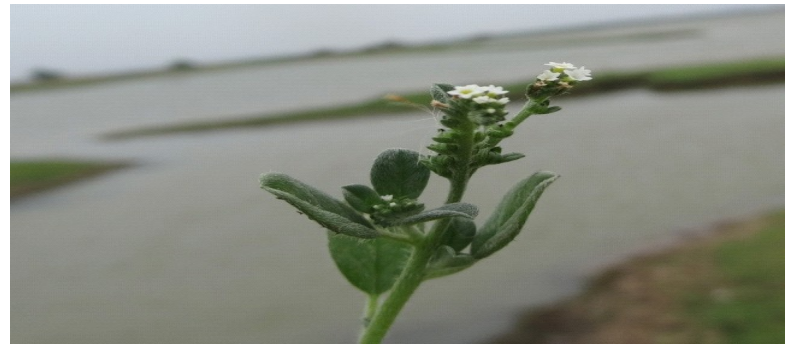

B) H eliotrophium curasavica

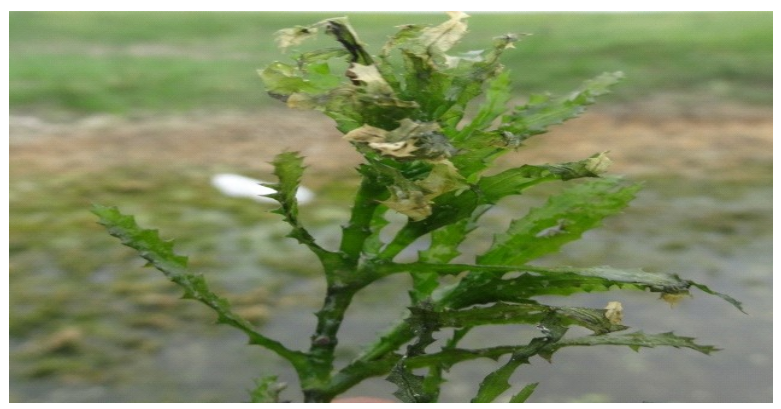

D) Najas indica (W illd.) Cha m

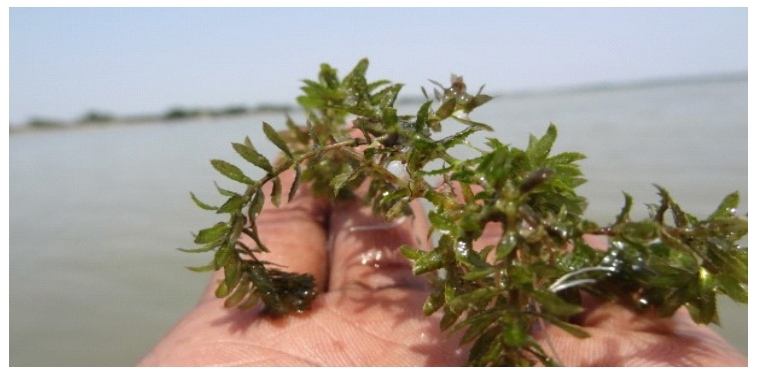

F) H ydrilla

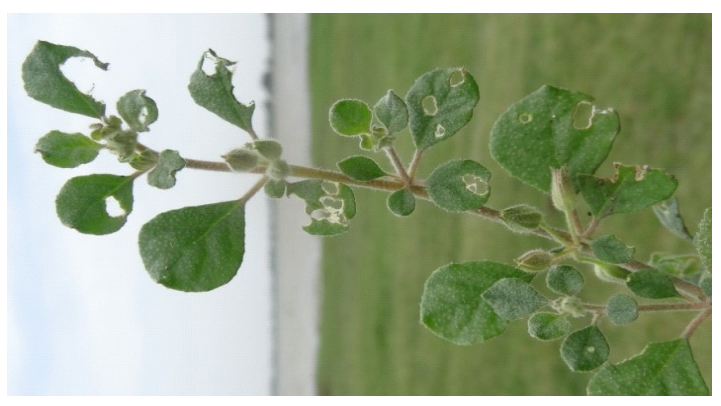

H) G linnus loto ides 
Macrophytes: plate. 2

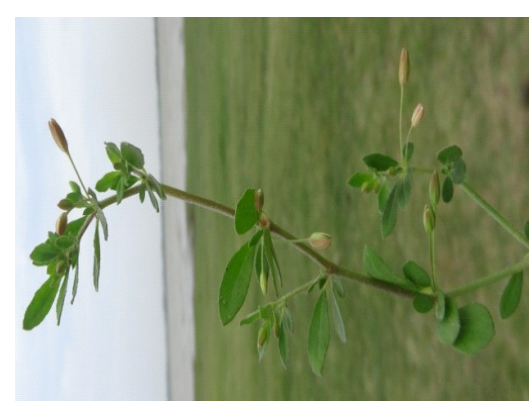

I) Goppositifolia

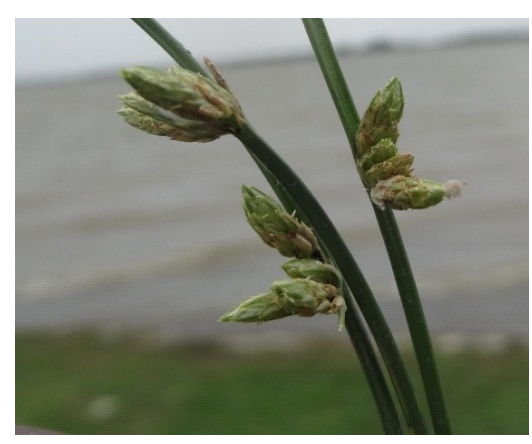

K) Scirpus articulatus $\mathrm{L}$.

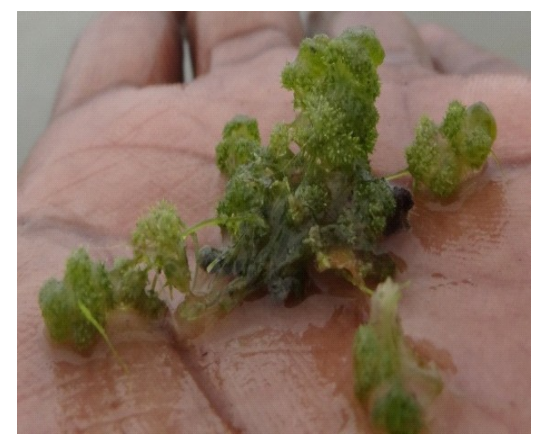

M) Nitella sp

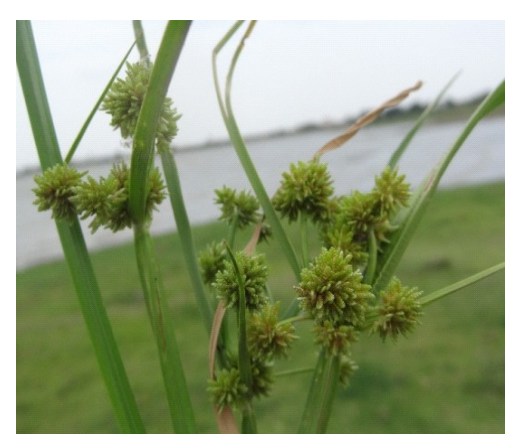

O) Cyperus corymbosus

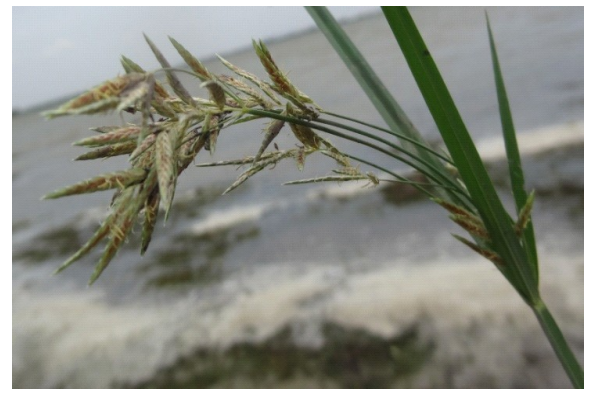

J) Cyperus arenarius Retz

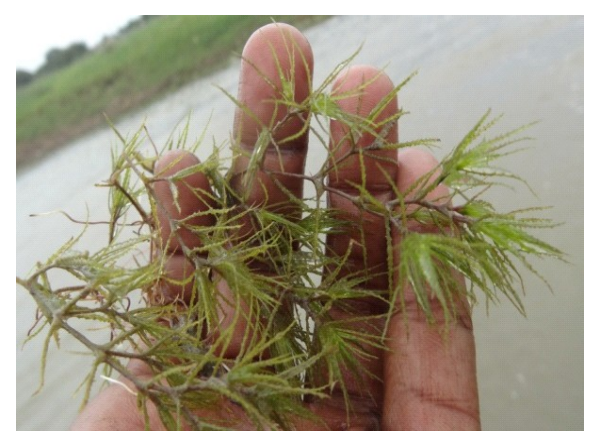

L) Ceratophyllum demersum $\mathrm{L}$

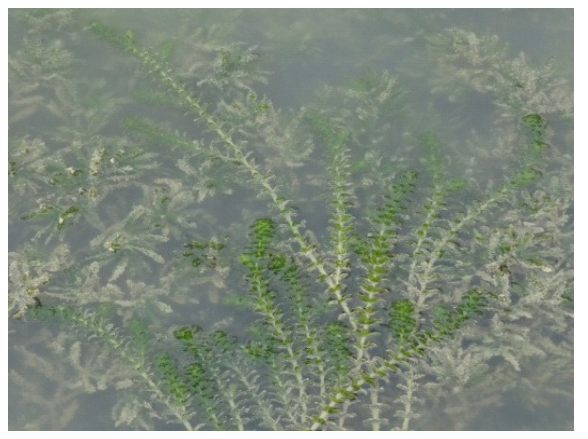

N) Hydrilla verticillata (L.f.) Royle.

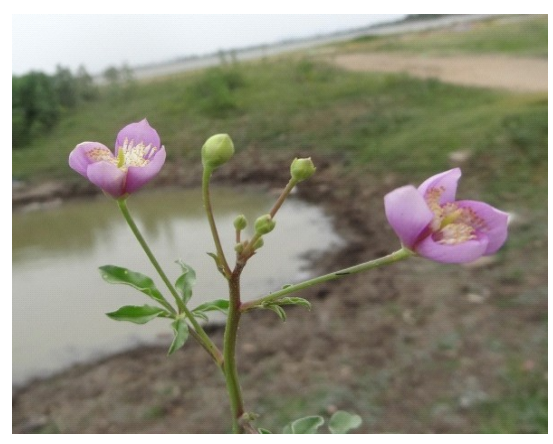

P) Cleome chelidoni 
Plate.3 Phytoplankton

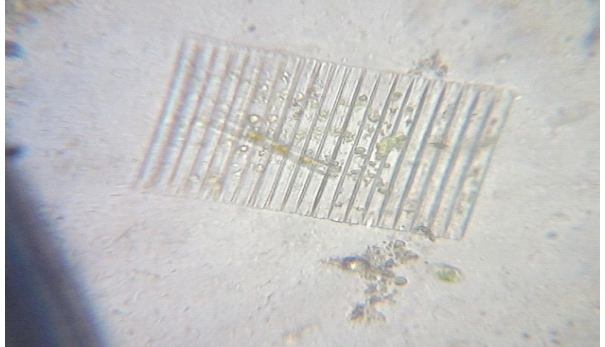

i) Fragillaria brevistriata Grun

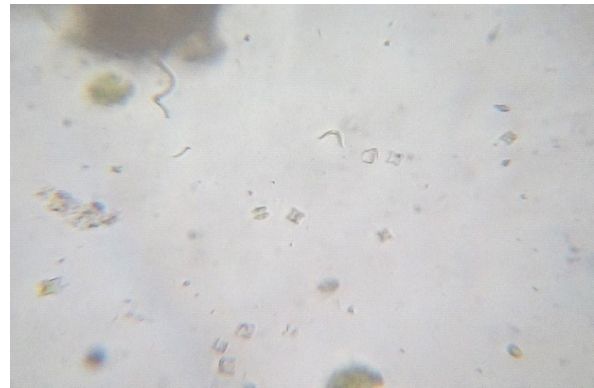

iii) Ankistrodesmus falcatus (Corda) Ralfs

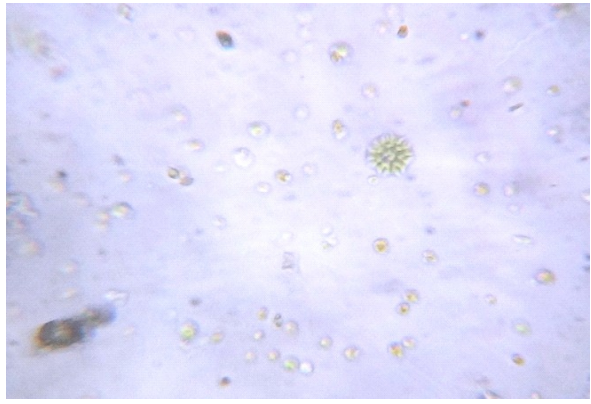

v) P.simplex

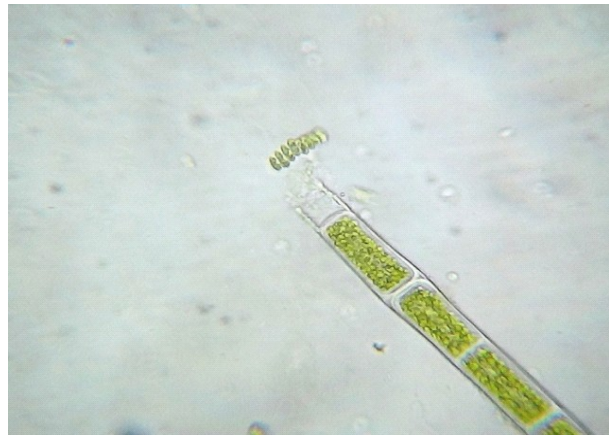

vii) Scenidesmus dimorphosus

Seasonal appearance of the prominent phytoplankton's of Kamalapur has also

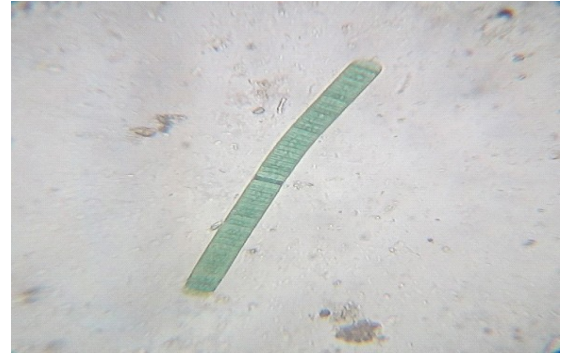

ii) Oscillatoria tenuis

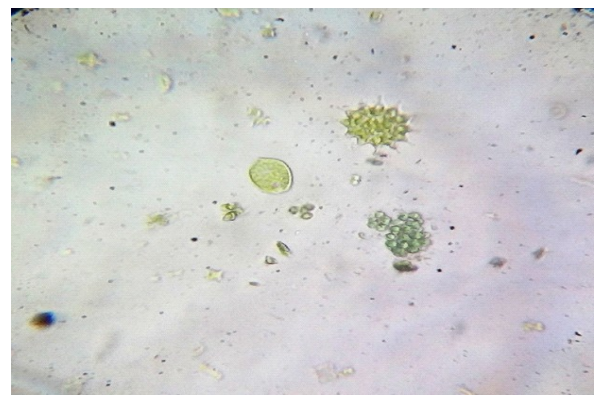

iv) Phacus acuminatus \& Pediastrum $\mathrm{sp}$

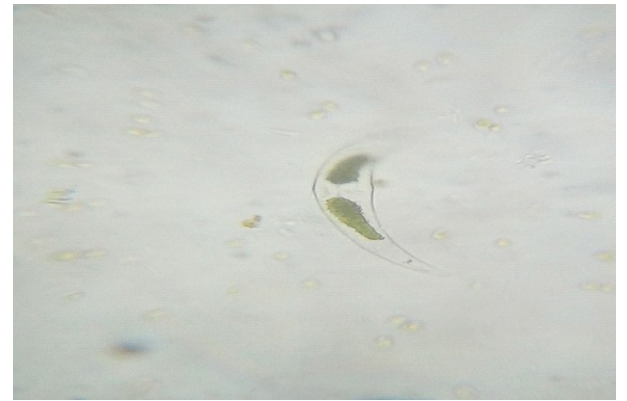

vi) Cosmarium botrytis

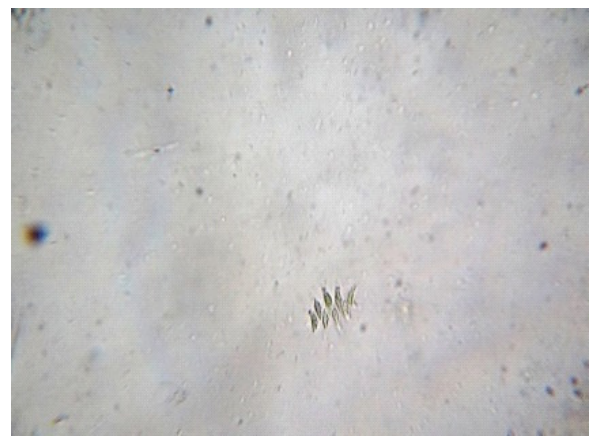

viii) S.quadracauda

been studied in which the two most prominent blue green algae Nostoc, Lyngbya 
$m$ two important diatoms, Pinnularia, Navicula and green-alga Spirogyra, Pithiphora, during the year 2013-2015. Of the two most prominent Cyanophyceae members Lyngbya was found to be dominant in March minimum in January. Nostoc has been shown to be dominant in February lowest in October. The Bacillariophyceae members were observed more dominantly in the month of March and the lowest in the month of September, maximum in March, with minimum in September. The green algae, Pithiphora was the only genus observed most dominantly in July least in the month of October under study. During the observation period between June 20013 - May 2015, the seasonal variation of all the members of Cyanophyceae, Bacillariophyceae and Chlorophyceae were found in almost all the seasons and in every month of 2013-2015. The phytoplankton populations of the reservoir varied with the seasonal variations and the maximum phytoplankton production coincided with the optimum water depth.

In conclusion, the macrophytic population decides the fish species composition and species richness. The water quality also decides by macrophytes documented by several authours earlier. As like this phytoplankton population also determines the zooplankton species. Bacillariophyceae algae are considered for water quality and organic pollution status by Palmer (1969). But due to anthropogenic activities causes even back ward area water resources also contaminated by organic pollution. The studied fresh water ecosystem was in good condition in the sense of organic pollution.

\section{Acknowledgement}

The author grateful thank to Prof. S. Seeta Ram Rao, Professor, Department of Botany, Plant Physiology And Molecular Biology
Lab.UCS, Osmania University, Hyderabad, Telangana.

\section{References}

Ambasht, R. S. (2005). Macrophytes limnology in the Indian subcontinent. Ukaaz Publication, Hyderabad: $58-174$

Anitha, G.S.,V.A.Chandrasekhar and M.S.Kodarkar 2005 Limnological studies on MIR Alam lake Hydrabad. Poll. Res. 24:681 - 687.

APHA 2012 Standard Methods for examination of water and wastewater (22nd ed.), 1175 pp. American Public Health Association, Washington DC.

Bentham, G. \& Hooker, J.D. 1862 - 1883. Genera Plantarum. 3-vols. L. Reeve \& Co Ltd, Ashford, Kent. London.

Bhaskar, V. \& Raji, B.A. 1973. Hydrophytes and marsh plants of Mysore city. Prasaranga, University of Mysore, Mysore, India

Bhatt, L. R., P. Lacoul, H. D. Lekhal and P. K. Jha 1999 Physico-chemical characteristic and phytoplanktons for Taudha lake, Kathmandu. Poll. Res. 18 (4): 353-358.

Bhattacharjee DK, Sarma SK, Bora PC, Kar A, Journal of Advance Plant Sciences, 2008, 4(1\&2), 69-73.

Bhattacharjee DK, Sarma SK, Devi B, Journal. Eco. Taxo.Bot, 2006, 30 (suppl), 133-139.

Billore DK, Vyas IN, International Journal of Ecological Science, 1981, (7), 4554.

Biswas K, Calder CC, Hand book of common water and marsh plants of India and Burma. Calcutta. 1936. (revised ed.1954).

Borah B, Sarma SK, Journal of Advance plant Sciences, 2012, 6 (5 \& 6), 91101. 
Carpenter, S. R., Lodge, D. M. (1986) Effects of submersed macrophytes on ecosystem processes. Aquatic Bot 26: 341-370.

Carpenter, S. R., N. F. Caraco, D. L. Correll, R. W. Howarth, A. N. Sharpley, and V. H. Smith 1998 Nonpoint pollution of surface waters with phosphorus and nitrogen. Ecological Applications. 8:559-568.

Chambers, P.A., P. Lacoul, K.J., Murphy, S.M., (2010). World checklist of macrophyte species.

Chaudhari A.M., Mahajan S.R. and Nandan S.N. 2007. Some Nostocaceae from paddy field soils of North Maharashtra, Research link-37(2); 13-15.

Cook, C.D.K. (1996). Aquatic and wetland plants in India Oxford University press. London.

Cowardian LM, Carter V, Golet FC, LaRoe ET, Classification of Wetlands and Deepwater Habitats of the United States. FWS/OBS-79/31. U.S. Fish and Wildlife Service: Washington, D.C., 1979.

Deka U, Sarma SK, New York Science Journal, 2014, 7 (6), 1-8.

Desikachany, T.V. 1959 Cyanophyta.686pp. ICAR Monograph, New Delhi. India.

Dhanalakshmi, V., K.Shanthi and K.M.Remia 2013 Physicochemical study of Eutrophic pond in Pollachi town, Tamilnadu, India. Int.J.Curr.Microbiol.App.Sci. 2013, 2(12): 219-227.

Dhande J.S. and Jawale A.K. 2006. On Oedogonium (Link) Hirn from Jalgaon District, Maharashtra, GEOBIOS 33(4); 321-322.

Dhande J.S. and Jawale A.K. 2007. On Spirogyra (Link) from Hartala lake, District Jalgaon, Maharashtra, Proceeding Nat. Symp. "Recent
Trends in Algal Biodiversity", 101103.

Dhande J.S. and Jawale A.K. 2008. Oedogonium (Chlorophyceae, Oedogoniales) from Jalgaon District, Indian Hydrobiology, 11(1), 43-46.

Dhande J.S. and Jawale A.K. 2008. On Genus Fragilaria lyngbye and Synedra Ehr. From Hartala lake, Maharashtra, Indian Hydrobiology, 11(2), 217-222.

Dhande J.S. and Jawale A.K. 2009. Genus Cosmarium corda from Hratala lake District Jalgaon Maharashtra, Shood samiksha aur Mulyacan,7, 196-198.

Dutta R, Barua D, Sarma SK, Hazarika LP, Nature Environment and Pollution Technology, 2010, 9(2), 283

Dutta R, Baruah B, Sarma SK, Annal of Biological Research, 2011, 2(4), 268-280.

Dutta, S.1985 The Mataks and Their Kingdom: Castes and Tribes of Assam. Chugh Publications. Assam (India), $279 \mathrm{pp}$.

Fokmare, A. K. and M. Musaddiq 2001 Comparative Studies of PhysicoChemical and Bacteriological Quality of Surface and Ground Water at Akole (MS). Pollution Research. 4(1): 56-61. J. Algal Biomass Utln. 2014, 5 (2): 1 - 7 Assessment of water quality using phytoplankton ISSN: 2229- 69055

Fritsch, F.E. 1961 The structure and the reproduction of the algae, Vol II. 791pp. University Press, Cambridge.

Fritsch, F.E.1935 The structure and the reproduction of the algae. Vol I. 791pp. University Press, Cambridge.

Ghosh SK, Illustrated Aquatic and Wetland Plants in Harmony with Mankind, Standard Literature, Kolkota,2005.

Goel, P.N., A. Y. Khatavkar, A. Y. Kulkarni and R. K. Trivedy 1986 
Limnological studies of a few freshwater bodies in southwestern Maharasthra with special reference to their chemistry and pollution. Poll. Res. 5 (2): 79-84.

Gopal B, Wetland and biodiversity: How to Kill Two Birds With One Stone ? In: W.Giesen (Ed.). Wetlands Biodiversity and Development. Proceeding of Workshop of the International Conference on Wetlands and Development held in Kuala Lumpur, Malaysia, and 913 October $1995 . \quad$ Wetlands Internationals, Kuala Lumpur, 1997,pp18-28.

Hulyal S.B. and B.B. Kaliwal 2011 Seasonal Variations in Physico-Chemical Characteristics of Almatti Reservoir of Bijapur district, Karnataka State. I.J.E.P. 1(1):58-67.

Jawale A.K, Kumawat D.A. and Chaudhari N.A. 2009. Fresh water Chlorophyceae from Jalgaon District, North Maharashtra IUnicellular Volvocales, Indian Hydrobiology, 12(1); 1-9.

Jawale A.K, Kumawat D.A. and Chaudhari N.A. 2009. Fresh water Chlorophyceae from Jalgaon District, North Maharashtra IIcolonial Volvocales, J. Indian bot. Soc., 88(3 \& 4); 231-235.Haranbaree dam and Mosam river of Maharastra. J. Environ. Biol. 26:223-227

Jawale A.K, Kumawat D.A. and Chaudhari N.A. 2010. Additions to the Volvocales Maharashtra I, Indian Hydrobiology, 13(1); 13-18.

Jawale A.K, Kumawat D.A. and Chaudhari N.A. 2010. Some members of order Chlorococcales new to Maharashtra, BIOINFOLET, 7(2); 94-97.

Jawale A.K, Kumawat D.A. and Chaudhari N.A. 2010. Some taxa of Chlamydomonas (Chlorophyceae:
Volvocales) new to Maharashtra, BIOINFOLET, 7(4); 298-301.

Jawale A.K, Kumawat D.A. and Dhande J.S. 2005. Desmids from fish ponds at Anjale District Jalgaon (M.S.) India, Proceeding National conference in Plant Science, Pravaranagar, 472478.

Jawale A.K. and Dhande J.S. 2005. A Preliminary survey of Chlorococales from Hartala lake - Genus Scenedesmus meyen, Plant Diversity and Biotechnology, 45-48.

Jawale A.K. and Dhande J.S. 2005. Some species of Oedogonium form Hartala Lake, District Jalgoan, Maharashtra, J. Aqua. Biol. Vol. 20(2), 17-20.

Kachroo, P. 1984. Aquatic Biology in India. Bishen Singh Mahendra Pal Singh, Dehra Dun

Kannan V. and S.V. Job 1980 Diurnal depth wise and seasonal changes of physicochemical factors in Sathio reservoir. Hydrobiol.70 :103-117.

Karr, J.R., J D. Allen, and A. C. Benke 2000 River conservation in the United States and Canada. In P. J. Boon, Davies and B.R. Petts, G E (Ed.), Global perspectives on River conservation, pp 3-39 Science, Policy, and Practice. Wiley, New York.

Kayode, J. and Ogunleye, O.T. (2008) Checklist and Status of Plant Species Used as Spices in Kaduna State of Nigeria. African Journal of General Agriculture 4, 13-18.

Khurshid, S. Zaheeruddin and A.Basheer 1997 Pollution assessment and water quality status in parts of Cochin. I.J.E.P.18(4):246-249.

Kiran, B.R., Patel A.N., Kumar Vijaya and Puttaiah E.T. (2006). Aquatic macrophytes in fish culture ponds at Bhadra fish farm, Karnataka. J. Aqua.Biol. 21(2): 27-30. 
Koshy, M. and T. V. Nayar 1999 Water quality aspects of river Pampa. Poll. Res. 18(4):501-510.

Kützing, F. T. 1895 Species Algarum VI (1) 922pp, Brockhaus, Leipzing.

Mahajan Neelama and Mahajan A.D. 1990. On some fresh water Blue green algae form Satpuda ranges in Jalgaon District (M.S.), Persectives in Phycology; 157-159.

Mahajan S.R. 2000. Saprobity system for the assessment of water quality of Velhala lake of Jalgaon, Maharashtra, J. Aqua. Biol. Vol. 17(1);1-4.

Mahajan S.R. and Nandan S.N. 2004. Blue green algae of Hartala lake of Jalgaon, Maharashtra, J.Aqua.Biol. 19(1): 11-12, 2004

Mahajan S.R. and Nandan S.N. 2005. Studies on algae of polluted lakes of North Maharashtra (INDIA), Plant diversity and Biotechnology, 67-71.

Mahajan S.R. and Nandan S.N. 2007. Contribution to the knowledge of Euglenoids of Hartala lake of Jalgaon, Maharashtra, Proc. Nat. Symp. "Recent trends in algal biotechnology and biodiversity" 110113.

Nandan S.N. and Mahajan S.R. 2006. Cyanobacterial diversity in polluted lakes of Jalgaon district of North Maharashtra, Aquatic Environment and toxicology, 28-61.

Nandan S.N. and Mahajan S.R. 2006. Studies on algae of polluted lakes of Jalgaon (Maharashtra): Role of Blue Green Algae, Ecology of lakes and Reservoirs, 54-62.

Niroula B., K.L.B. Singh, G.B. Thapa and J. Pal 2010 Seasonal Variations in Physico-Chemical Properties and Biodiversity in Betana Pond, Eastern Nepal. Our Nature. 8: 212-218.

Palmer, C.M. 1969 Composite rating of algae, tolerating organic pollution. British Phycology Bullettin.5:78-92.

Palmer, C.M.1959 Algae in water supplies, US Department of Health, Education and Welfare, Public Health Service, Cincinnati. (Public Health Service publication No. 657).

Panigrahi, S.N., B.B. Nayak and B.C.Acharya 2001 Plankton algae as pollution index of Maipura estuary, east coast of India. J.mar.biol.Ass.Indi. 43(1\&2): 168172.

Pathak, H.,D. Pathak and S. N. Limaye 2012 Studies on the physico-chemical status of two water bodies at Sagar city under anthropogenic Influences. Advances in Applied Science Research. 3 (1):31-44.

Prasad, B.N., Y.C. Jaitly and Y. Singh 1985 Periodicity and interrelationships of physicochemical factors in pond. Proc. Nat. Symp. Pure and Applied Limnology (ed Adoni A.D.) Bull. Bot.Soc. Sagar. 32: 1-11.

Prescott, G.W. 1975 Algae of the Western Great Lake area. 977pp. W Mc Brown company publishers, Iowa.

Radhika, C. G., I. Mini and T. Gangadevi 2004 Studies on abiotic parameters of a tropical fresh water lake Vellayani Lake, Trivandrum, Kerala. Poll. Res 23(1):49-63.

Ramulu N. K. and G. Benarjee 2013 Physicochemical factors influenced plankton biodiversity and fish abundance- A case study of Andhra Pradesh. Int. J. Lifesc. Bt. \&Pharm. Res.1(2):248-260.

Saha, S. B., S. B. Bhattacharya and A Choudhury 2000 Diversity of phytoplankton of sewage pollution brakish water tidal ecosystems. Environ. Biol. 21 (1): 9-14.

Shekhar, S., B.R.Kiran, E.T. Puttaiah, Y. Shivaraj and K.M. Mahadevan 2008 
Phytoplankton as index of water quality with reference to industrial pollution. J. Environ. Biol. 29(2): 233-236.

Smith, G.M. 1950 The freshwater Algae of the United States 719pp. Mc. Grew Hill book company, Inc. NY. Toronto.

Smitha, V.H., G.D. Tilmanb and J.C. Nekolac 1999 Eutrophication: impacts of excess nutrient inputs on freshwater,marine, and terrestrial ecosystems. Environmental Pollution. 100:179-196.

Stevenson, R. J. and Y. Pan 1999 Assessing environmental conditions in Rivers and streams using diatoms. In E. F.

Stoermer and J. P. Smol, (Ed.), The diatoms, Applications for the environmental and earth sciences, pp. 11-40 Cambridge University Press, Cambridge.
Thomas, S., P. Cecchi, D. Corbin and J. Lemoalle 2000 The different primary producers in a small African tropical reservoir during a drought: temporal changes and interactions. Freshwat. Biol. 45: 43-56.J. Algal Biomass Utln. 2014, 5 (2): 1 - 7

Trivedy, R.K. and P.K.Goel 1986 Chemical and biological methods for water pollution studies $248 \mathrm{pp}$. EnvironmentalPublications, Karad, India.

Verma, P.U., A. R. Purohit and N. J. Patel 2012 Pollution Status of Chandlodia Lake Located in Ahmedabad Gujarat,IJERA. 2:1600-1606.

Yadav, P., V. K. Yadav,, A.K. Yadav and P.K. Khare 2013 Physico-Chemical Characteristics of a Fresh Water Pond of Orai,U. P., Central India. Octa. J. Biosci. Vol. 1(2): 177-184.J. Algal

\section{How to cite this article:}

Odelu, G., 2016. Phytoplankton and Macrophytic Floral Studies in Kamalapur Reserviour of Karimnagar District of Telangana, India. Int.J.Curr.Microbiol.App.Sci.5(1): 78-94. doi: ihttp:///dx.doi.org/10.20546/jijomas. 2016.501 .007 